\title{
The immunological characteristics and probiotic function of recombinant Bacillus subtilis spore expressing Clonorchis sinensis cysteine protease
}

Zeli Tang ${ }^{1,2 \dagger}$, Mei Shang ${ }^{1,2 \dagger}$, Tingjin Chen ${ }^{1,2}$, Pengli Ren ${ }^{1,2}$, Hengchang Sun ${ }^{1,2}$, Hongling Qu ${ }^{1,2}$, Zhipeng Lin ${ }^{1,2}$, Lina Zhou ${ }^{1,2}$, Jinyun $Y u^{1,2}$, Hongye Jiang ${ }^{1,2}$, Xinyi Zhou ${ }^{1,2}$, Xuerong Li $i^{1,2}$, Yan Huang ${ }^{1,2}$, Jin $X^{1,2}$ and Xinbing $Y u^{1,2^{*}}$

\begin{abstract}
Background: Clonorchiasis, a food-borne zoonosis, is caused by Clonorchis sinensis. The intestinal tract and bile ducts are crucial places for $C$. sinensis metacercariae to develop into adult worms. The endospore of Bacillus subtilis is an ideal oral immunization vehicle for delivery of heterologous antigens to intestine. Cysteine protease of $C$. sinensis (CsCP) is an endogenous key component in the excystment of metacercariae and other physiological or pathological processes.
\end{abstract}

Methods: We constructed a fusion gene of CotC (a coat protein)-CsCP and obtained B. subtilis spores with recombinant plasmid of pEB03-CotC-CsCP (B.s-CotC-CSCP). CotC-CsCP expressed on spores' surface was detected by Western blotting and immunofluorescence. Immunological characteristics of recombinant spore coat protein were evaluated in a mouse model. The levels of CsCP-specific antibodies were detected by ELISA. Effects of recombinant spores on mouse intestine were evaluated by histological staining. The activities of biochemical enzymes in serum were assayed by microplate. Liver sections of infected mice were evaluated by Ishak score after Masson's trichrome.

Results: The B.s-CotC-CsCP spores displayed CsCP on their coat. Specific lgG and isotypes were significantly induced by coat proteins of B.s-CotC-CsCP spores after subcutaneous immunization. IgA levels in intestinal mucus and bile of B.s-CotC-CSCP orally treated mice significantly increased. Additionally, more IgA-secreting cells were observed in enteraden and lamina propria regions of the mouse jejunum, and an increased amount of acidic mucins in intestines were also observed. There were no significant differences in enzyme levels of serum among groups. No inflammatory injury was observed in the intestinal tissues of each group. The degree of liver fibrosis was significantly reduced after oral immunization with B.s-CotC-CsCP spores.

Conclusions: Bacillus subtilis spores maintained the original excellent immunogenicity of CsCP expressed on their surface. Both local and systemic specific immune responses were elicited by oral administration of B.s-CotC-CsCP spores. The spores effectively promoted intestinal health by inducing secretion of acidic mucins, with no other side effects to the liver or intestine. Oral administration of spores expressing CSCP could provide effective protection against C. sinensis. This study may be a cornerstone for development of antiparasitic agents or vaccines against clonorchiasis based on $B$. subtilis spore expressing CSCP on the surface.

Keywords: Clonorchis sinensis, Bacillus subtilis spore, Cysteine protease, Oral immunization, Immunological characteristics

\footnotetext{
* Correspondence: yuhxteam@163.com

'Equal contributors

'Department of Parasitology, Zhongshan School of Medicine, Sun Yat-sen

University, 74 Zhongshan 2nd Road, Guangzhou 510080, China

${ }^{2}$ Key Laboratory for Tropical Diseases Control, Sun Yat-sen University, Ministry

of Education, Guangzhou 510080, Guangdong, China
} 


\section{Background}

Clonorchiasis caused by Clonorchis sinensis is recognized as an important emergent or re-emergent human foodborne parasitic disease, one of the most common zoonoses in East Asia. Humans or animals can be infected mainly due to ingestion of raw or undercooked freshwater fish containing encysted metacercaria of $C$. sinensis $[1,2]$. Metacercariae excyst in the duodenum of the host, then migrate into the bile duct, and further develop into adult worms [2]. Mechanical irritation, immunopathological processes and DNA damage caused by $C$. sinensis can induce hyperplasia of the bile duct epithelium and connective tissue and cause jaundice, indigestion, biliary inflammation and bile duct obstruction, even cholangiocarcinoma (CCA), liver cirrhosis and liver cancer in humans $[1,3]$. Clonorchiasis has become a severe disease burden and brought serious medical and economic problems to the low- or middle-income countries of East Asia. It is estimated that more than 200 million people are threatened by $C$. sinensis infection, and over 15 million people are infected worldwide [2, 4]. The global burden of clonorchiasis is nearly 275,370 disability adjusted life years (DALYs), and 5,591 people have died from this infection every year [4]. It is urgent that effective prevention strategies such as vaccine trials, the development of antiparasitic agents and new health education be implemented.

Improvement of mucosal immunity is very important in conferring protection against pathogens (e.g. internal parasites) that typically invade via mucosal system $[5,6]$. Oral immunization, for example, is a very straightforward, inexpensive and needle-free approach to deliver a vaccine to the mucosal lining of the gut and elicit protective immunity within the gut mucosa. However, oral immunization suffers from degradation by gastric acid and proteolysis in gastrointestinal tract, which generates a poor immune response [6,7]. Therefore, effective heterologous antigen carriers should be chosen to solve the problem of limited absorption and tolerance in the gut.

A series of reports indicated that the endospore of Bacillus subtilis is an ideal vehicle for delivery of heterologous antigens to the gastrointestinal tract. First, spore-forming B. subtilis is a non-pathogenic and noninvasive aerobic Gram-positive bacterium [8, 9]. Spores of various Bacillus species are currently being used as probiotics and food supplements in both humans and animals $[9,10]$. They can survive under extreme temperature, desiccation, $\mathrm{pH}$ and exposure to noxious chemicals and solvents $[11,12]$. In addition, these spores possess convenient gene operability. Heterologous antigens can be stably and sufficiently displayed on the surface of spores using the outer coat proteins of the B. subtilis spore (such as $\operatorname{Cot} B, \operatorname{Cot} C$ and $\operatorname{Cot} G$ ) as the fusion partner $[12,13]$. In our laboratory, the oral immunization delivery platform based on a B. subtilis spore-engineering system has been successfully constructed and is proven to be valid and feasible [14-16].

The cysteine protease superfamily of parasite organisms plays a key role in physiology and related pathobiology processes that are closely related to larval migration, nutrition acquisition, egg hatching and immune evasion [3, 17-19]. Currently, cysteine proteases identified from various parasites (e.g. C. sinensis, Fasciola hepatica, Taenia solium and Ancylostoma caninum) have been exploited as major potential molecules for serodiagnosis, immuno-/chemo-therapy and vaccine candidates [19-25]. The cysteine protease from $C$. sinensis $(\mathrm{Cs} \mathrm{CP})$ is an important component of excretorysecretory products (ESPs) in both adult worms and metacercariae $[19,26]$. Additionally, $C s C P$ is an indispensable protease participating in the excystment of metacercariae, enabling larvae to successfully move to bile ducts, and further develop into adults [26]. Therefore, CsCP will be a potential target for effective vaccines and drug development.

In the present report, the encoding sequence of $\mathrm{Cs} C \mathrm{P}$ was transformed into B. subtilis (WB600) using an Escherichia coli/B. subtilis shuttle vector. The CotC$\mathrm{CsCP}$ fusion gene was constructed and expressed on spore coat. Mice were administered subcutaneously with coat proteins extracted from CotC-CsCP-transformed $B$. subtilis (B.s-CotC-CsCP) spores or recombinant $C s \mathrm{CP}$ protein purified from an $E$. coli expression system $(\mathrm{r} C s \mathrm{CP})$. The evoked immune reactions were compared. Additionally, the specific local and systemic immune responses triggered by B.s-CotC-CsCP spores were analysed, as were the effects on the intestinal environment in mice after oral administration of B.s-CotCCsCP spores.

\section{Methods \\ Expression and purification of $\mathrm{rCsCP}$}

The coding sequence of CsCP (accession number JN655695), minus the signal sequence, was amplified from a $C$. sinensis cDNA library and cloned into the pET-28a (+) vector. The recombinant plasmid was transformed into E. coli BL21 (DE3) (BL21-pET28a$C s \mathrm{CP}$, abbreviated as $\mathrm{BL} 21-\mathrm{Cs} \mathrm{CP}$ ) and induced by isopropyl $\beta$-D-thiogalactopyranoside (IPTG) as described previously [19]. The induced E. coli was collected by centrifugation and ultrasonicated to obtain inclusion bodies in sediment. The inclusion bodies were dissolved in phosphate-buffered saline (PBS) containing 2, 4 and $6 \mathrm{M}$ urea, and $\mathrm{rCs} \mathrm{CP}$ was released into the supernatant. Finally, rCsCP was purified with the His Bind Purification kit (Novagen, Darmstadt, Germany), and gradient elution with 5-400 mM imidazole [15, 19]. 


\section{The construction of B.s-CotC-CsCP}

The coding sequence of coat protein $C(\operatorname{Cot} C)$ from $B$. subtilis spores, a protein over-expressed on spore surface, was amplified by polymerase chain reaction (PCR) using specific primers (F: 5'-CAT GTC GAC TGT AGG ATA AAT CGT T-3', R: 5'-CGG AAG CTT GTA GTG TTT TTT ATG C-3', where the underlined portions are restriction sites for Sal I and Hind III). The DNA sequence was inserted into the multiple clone site of the pBluescript II SK (-) plasmid [15, 27] after digestion with Sal I and Hind III. The coding sequence of $C s C P$ was amplified from the previously constructed pET28a $(+)-C s C P$ plasmid using specific primers (F: 5'-CCC AAG CTT AGC AAC ATT CCT GAA TCA G-3', R: 5' - TAC GAG CTC TCA CAA GAT GAT CGA GGT G-3') containing restriction sites for Hind III and Sac I (underlined). The sequence was inserted into pBluescript II SK (-)-CotC plasmid, followed by CotC using Hind III and Sac I restriction sites, and transformed into E. coli DH5 $\alpha$ (Promega, Madison, USA). Finally, the CotC- $C s \mathrm{CP}$ fusion gene was sub-cloned into a pEB03 shuttle vector after digesting with Sal I and Sac I. The recombinant plasmid of pEB03-CotC-CsCP was colony-formed in DH5a and easily electro-transformed into B. subtilis WB600 to construct B.s-CotC-CsCP. Similarly, B. subtilis WB600 with the pEB03-CotC plasmid (B.s-CotC) was obtained by electro-transformation using specific primers (F: $5^{\prime}$ CAG AAG CTT TGT AGG ATA AAT CGT T-3', R: 5'-CGC GAG CTC TTA GTA GTG TTT ATG C-3', restriction sites underlined). All recombinant plasmids above were confirmed by sequencing.

\section{Preparation of spores and coat proteins}

Bacillus subtilis WB600 with pEB03-CotC-CsCP was cultured in Difco Sporulation Medium (DSM, BD, Franklin Lakes, USA), and the spores were obtained by the exhaustion method as described previously [28]. After incubation for $24 \mathrm{~h}$, the spores were harvested by centrifugation, treated with $1 \mathrm{mM}$ phenylmethylsulfonyl fluoride (PMSF) plus $4 \mathrm{mg} / \mathrm{ml}$ lysozyme for $30 \mathrm{~min}$ at room temperature (RT), washed with $1 \mathrm{M} \mathrm{NaCl}$ and $1 \mathrm{M} \mathrm{KCl}$, and washed twice with distilled water. Finally, the spores were re-suspended in sterile water and treated in a $68{ }^{\circ} \mathrm{C}$ water bath for $1 \mathrm{~h}$ to kill the residual WB600 propagules. To extract the coat proteins of spores, the induced spores were treated with sodium dodecyl sulfate (SDS)-dithiothreitol (DTT) extraction buffer $(0.5 \%$ SDS, $0.1 \mathrm{M} \mathrm{DTT}, 0.1 \mathrm{M} \mathrm{NaCl})$ at $37{ }^{\circ} \mathrm{C}$ for $2 \mathrm{~h}$, washed with $1 \mathrm{M}$ Tris- $\mathrm{HCl}$ buffer $(\mathrm{pH}$ 8.0) six times, and suspended in $5 \mathrm{ml}$ broken buffer $(50 \mathrm{mM}$ Tris- $\mathrm{HCl}, 0.5 \mathrm{mM}$ EDTA, $1 \mathrm{mM}$ PMSF) and ultrasonicated for $5 \mathrm{~min}$. Coat proteins were collected from the precipitate after centrifugation.
SDS-PAGE, Western blotting and mass spectrometry analysis The spores induced at different time points $(0 \mathrm{~h}, 6 \mathrm{~h}, 12 \mathrm{~h}$ and $24 \mathrm{~h}$ ) and the precipitate/supernatant of the sonicated lysates were analysed by $12.0 \%$ SDS-polyacrylamide gel electrophoresis (SDS-PAGE) to evaluate the expression of the $\operatorname{CotC}-\mathrm{Cs} C \mathrm{P}$ fusion protein. The separated proteins were transferred onto polyvinylidene fluoride (PVDF) membranes for Western blotting analysis. The membranes were blocked with $5 \%$ non-fat milk in phosphate buffer saline containing $0.05 \%$ Tween 20 (PBS-T) for $2 \mathrm{~h}$ at RT and incubated with rat anti-r $C s C P$ serum (1:600) overnight at $4{ }^{\circ} \mathrm{C}$. After washing three times in PBS-T, the membranes were incubated with HRP-conjugated rabbit anti-rat IgG (1:2000, Sigma, St. Louis, USA) for $1 \mathrm{~h}$ at RT and were detected by enhanced chemiluminescence (ECL). Meanwhile, the induced spores containing pEB03-CotC-CsCP or pEB03-CotC, BL21 with pET-28a-CsCP and purified $\mathrm{rCs} C \mathrm{P}$ from BL21 were comparatively analysed by $12 \%$ SDS-PAGE. The corresponding bands of purified $\mathrm{rCs} C \mathrm{P}$ and $\mathrm{CotC}-\mathrm{Cs} \mathrm{CP}$ fusion protein in the polyacrylamide gel were cut out, digested with trypsin and injected onto an ABI 4800 Proteomics Analyzer Matrix-assisted laser desorption ionization time-of-flight mass spectrometer (MALDI-TOF-MS/MS) and Mascot search engine (Matrix Science, London, UK) for protein identification.

Immunofluorescence and fluorescent confocal microscopy To confirm whether CsCP displayed on the surface of spores, $200 \mu \mathrm{l}$ of the treated sporulation suspension was fixed onto slides according to improved methods previously described [14, 15]. Samples were blocked with normal goat serum for $2 \mathrm{~h}$ at RT and incubated with rat anti-rCsCP serum (1:200) overnight at $4{ }^{\circ} \mathrm{C}$. Following three PBS washes, Cy3-labeled goat anti-rat IgG (1:400, Invitrogen, Carlsbad, USA) was used to cover the samples smears for $1 \mathrm{~h}$ at RT (in dark). The samples were incubated for 3-5 min with a 4', 6-diamidino-2-phenylindole-(DAPI)-DNA staining solution. Fluorescent images were captured using a Leica DMI4000B fluorescent microscope and a ZEISS LSM 510 META confocal microscope. The induced spores containing pEB03-CotC served as the control.

\section{Immunogenicity analysis of subcutaneously immunized rCsCP and B.s-CotC-CsCP}

Female BALB/c mice aged 6-8 weeks were purchased from the Animal Center of Sun Yat-Sen University and raised carefully in accordance with the animal care and the ethical guidelines of the National Institutes of Health.

Each BALB/c mouse was immunized subcutaneously (s.c.) with $100 \mu \mathrm{g} \mathrm{rCsCP}$ or the coat proteins of the B.sCotC-CsCP spore, which were emulsified with complete Freund's adjuvant (Sigma-Aldrich, St. Louis, USA) at the first injection. Control mice were similarly administered 
with an equal volume of PBS plus adjuvant. Two booster injections at 2-week intervals were performed with $50 \mu \mathrm{g}$ of proteins or an equal volume of PBS emulsified in incomplete Freund's adjuvant (Sigma-Aldrich, St. Louis, USA). Anti-sera were collected every 2 weeks for 4 weeks (Fig. 1a) after the final boosting, and antibody titres were determined by ELISA. All anti-sera samples were split and stored at $-80^{\circ} \mathrm{C}$.

\section{Animals, oral immunization and sample collection}

Sixty mice were randomly and evenly divided into the following four groups as B.s-CotC-CsCP, BL21-CsCP, B.s-CotC and PBS. Mice were administered orally by gavage with spores $\left(1 \times 10^{9}\right)$, BL21-CsCP (equivalent CsCP as expressed by the spores), and PBS in a volume of $0.20 \mathrm{ml}$. Mice were treated three times in total (3 days each time) at biweekly intervals (Fig. 1b).

Serum samples were collected on days 5, 7, 10 and 14 after each administration. Mice were sacrificed two weeks after each oral treatment. The small intestines were isolated and washed 2-3 times with $0.4 \mathrm{ml}$ sterile PBS. The residual mucus was scraped using tweezers. Gallbladders were also collected. The midsection of mouse jejuna (approximately 5-7 mm) was excised and immersed in Bouin's solution (75\% saturated picric acid solution, 25\% formalin and 5\% glacial acetic acid) for $24 \mathrm{~h}$ before histological analysis.

\section{Detection of CsCP-specific antibodies by indirect ELISA}

The levels of IgG, IgG1 and IgG2a in serum were measured by an ELISA assay as described previously [15, 27].
Plates were coated with $5 \mu \mathrm{g} / \mathrm{ml}$ purified $\mathrm{rCsCP}(0.05 \mathrm{M}$ carbonate-bicarbonate buffer, $\mathrm{pH}$ 9.6) at $100 \mu \mathrm{l}$ per well and incubated at $4{ }^{\circ} \mathrm{C}$ overnight. After washing with PBS$\mathrm{T}$ three times, the plates were blocked with $5 \%$ skimmed milk for $2 \mathrm{~h}$ at $37{ }^{\circ} \mathrm{C}$. After washing routine program, the plates were incubated with serum samples (1:100) for $2 \mathrm{~h}$ at $37^{\circ} \mathrm{C}$ and subsequently with HRP-conjugated goat antimouse IgG (1:2000, Santa Cruz, USA), IgG1 (1:2000, Santa Cruz, USA), or IgG2a (1:2000, Invitrogen, Carlsbad, USA), respectively, for $1 \mathrm{~h}$ at RT. Next, $100 \mu \mathrm{l}$ tetramethylbenzidine (TMB, BD, Franklin Lakes, USA) solution was added to the plates for $10-15 \mathrm{~min}$ and stopped by $50 \mu \mathrm{l}$ of $2 \mathrm{M}$ $\mathrm{H}_{2} \mathrm{SO}_{4}$, and the absorbance was detected at $450 \mathrm{~nm}$. Before changing the incubation solution, the plates were washed with PBS-T 3 times.

Similarly, IgA levels in sera (1:20), intestinal lavage mucus (1:20), and bile (1:100) were also detected. HRP-conjugated goat anti-mouse IgA (1:2000, Southern Biotech, Birmingham, USA) was employed as a secondary antibody.

\section{Challenge infections and protective efficacy evaluation} Metacercariae of $C$. sinensis were isolated from Pseudorasbora parva, captured in Shuangfeng county, Hunan, as previously described [14, 15]. Two weeks after final booster, orally immunized mice ( $n=5$ for each group) were challenged with 30 living $C$. sinensis metacercariae through intragastric administration. All mice were sacrificed at 6 weeks post-challenge infection. Liver tissues were isolated and submitted to histopathological staining.

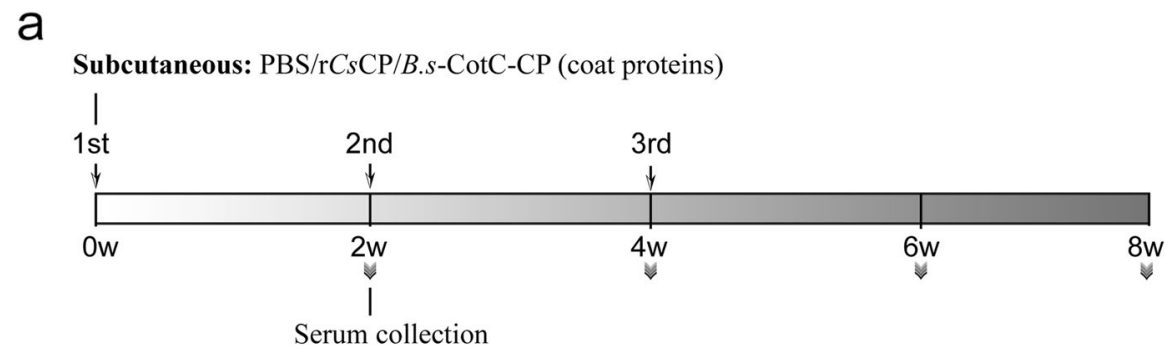

b

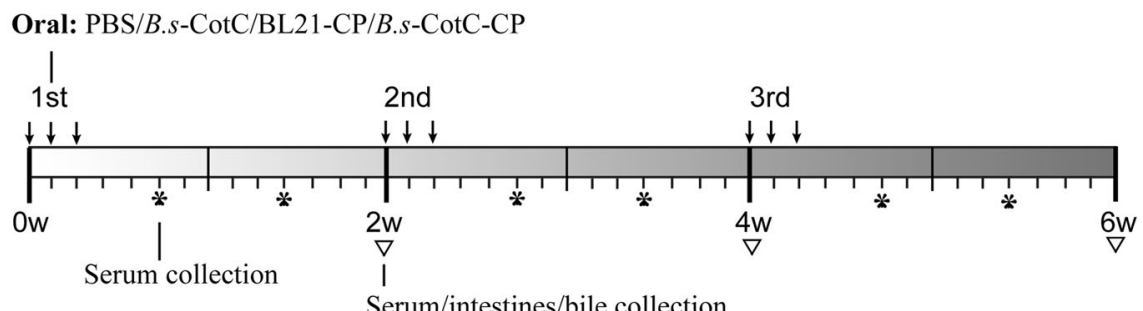

Fig. 1 Schematic of the treatment regimen. a Subcutaneous immunization of mice with emulsified PBS, $\mathrm{rCs} C \mathrm{C}$ or spore coat proteins of B. s-CotCCsCP administered three times. Serum samples were collected at 2, 4, 6 and 8 weeks. $\mathbf{b}$ Oral administration of mice with PBS, spores of B.s-CotC or B.s-CotC-CP, or BL21-CP three times in total, with continuous gavage for three days each time. Serum, intestine and bile samples were collected every 2 weeks. Additionally, serum samples were collected on days 5 and 10 after each administration. Abbreviations: B.s-CotC-CP, WB600 containing pEB03-CotC-CsCP; B.s-CotC, WB600 harbouring pEB03-CotC; BL21-CP, BL21 harbouring pET28a-CsCP 


\section{Histology staining}

After incubation with Bouin's solution, mouse jejuna from each group were dehydrated, embedded in paraffin wax and sliced into $5-\mu \mathrm{m}$ sections. The sections were rehydrated with xylene and a gradient ethanol, followed by PBS-T washing. After paraffin removal, the endogenous peroxidase sections were removed by using 3\% hydrogen peroxide. The sections were soaked in $0.01 \mathrm{M}$ citrate buffer to retrieve the antigens by high-pressure boiling for $10 \mathrm{~min}$. The slides were blocked with normal goat serum for $1 \mathrm{~h}$ at $\mathrm{RT}$, and incubated with goat antimouse IgA (1:600, Southern Biotech, Birmingham, USA) overnight at $4{ }^{\circ} \mathrm{C}$. After washing three times with PBS, the slides were incubated with HRP-Protein A (1:4000, Invitrogen, Carlsbad, USA) at RT for $1 \mathrm{~h}$. The immunoreactive signal was developed using 3, 3'-diaminobenzidine (DAB, ZSGB-BIO, China). Finally, the slides were stained with Mayer's haematoxylin, dehydrated and mounted with neutral balsam. Images were captured under a light microscope (Carl Zeiss, Germany).

For pathological observations, the jejunum sections were stained with routine haematoxylin and eosin (HE). Liver tissue sections from $C$. sinensis metacercariae infected mice were performed with routine Masson staining. All specimens were viewed under a light microscope (Carl Zeiss, Germany). Liver fibrosis of each group was morphologically evaluated by using the Ishak fibrosis score [29].

The mucus production of jejunum was evaluated using alcian blue-periodic acid Schiff reagent (AB-PAS, Baso, China) according to the manufacturer's protocol. Mucins were observed under a light microscope (Carl Zeiss, Germany).

\section{Detection of biochemical indices}

The serum of each group was collected at week 6 . Glutamic pyruvic transaminase/alanine aminotransferase (GPT/ALT) and glutamic oxaloacetic transaminase/aspartate aminotransferase (GOT/AST) were measured using an alanine aminotransferase assay Kit and an aspartate aminotransferase assay Kit, respectively (Jiancheng, China).

\section{Statistical analysis}

Experimental data were presented as the mean \pm standard deviation (SD) values. Student's $t$-test was performed to determine significant differences between groups using SPSS software 13.0 software. $P$-values $<0.05$ were considered as statistically significant.

\section{Results}

Expression and identification of fusion/recombinant protein The recombinant plasmids pEB03-CotC and pEB03CotC-CsCP were successfully constructed and sub-cloned into WB600 (Additional file 1: Figure S1). The $\mathrm{rCsCP}$ protein was overexpressed in IPTG-induced BL-21 containing pET28a-CsCP, and purified with gradient imidazole (Additional file 1: Figure S1, Additional file 2: Figure S2).

The molecular mass of the CotC-CsCP fusion protein was approximately $43.8 \mathrm{kDa}$, corresponding to the molecular mass of $C s \mathrm{CP}(35 \mathrm{kDa})$ plus $\operatorname{CotC}(8.8 \mathrm{kDa})$. As shown in Fig. 2, the CotC-CsCP fusion protein abundantly expressed in the DSM medium induced B.s-CotC-CsCP spores, while no matching band was observed in the medium of B.s-CotC spores. The expression of the fusion protein gradually increased with incubation time (Fig. 2b). In addition, the spore coat proteins contained large amounts of CotC-CsCP in the precipitation extract but almost no corresponding band was present in the supernatant (Fig. 2c). Western blotting analysis using rat anti$\mathrm{rCs} \mathrm{CP}$ serum as the primary antibody showed specific bands at $43.8 \mathrm{kDa}$ in spores samples incubated for $6 \mathrm{~h}$, $12 \mathrm{~h}$ and $24 \mathrm{~h}$ as well as for coat protein precipitation; however, no bands were observed in propagules and supernatant (Fig. 2f, g).

The mass spectrum analysis of $\mathrm{CotC}-\mathrm{Cs} \mathrm{CP}$ and $\mathrm{rCs} \mathrm{CP}$ is shown in Fig. 2. Both proteins were identified as the cysteine protease of $C$. sinensis and belonged to ProCathepsin L (Table 1).

\section{Immunofluorescence analysis of CsCP expressed on the spore surface}

Using the rat anti-rCsCP serum as the primary antibody and followed by Cy3-labelled goat anti-rat IgG as the secondary antibody, immunofluorescence and fluorescent confocal microscopy showed that red fluorescence abundantly distributed surrounded the spores' coat of B.s-CotC-CsCP with a high positive rate (Fig. 3a, b). Almost no red fluorescence was visualized on the B.s-CotC spores surface (Fig. 3c).

\section{Specific lgG and isotypes in serum of subcutaneous immunized mice}

The $\mathrm{r} C s \mathrm{CP}$ protein provoked markedly higher production of specific IgG compared with the control group only 2 weeks after the first immunization $\left(t_{(2)}=14.72, P=0.005\right)$. The significantly higher levels of anti-rCsCP IgG continued rising until four weeks after the last immunization $\left(t_{(2)}=\right.$ 351.27, $P=10^{-9}$ ). The titre of anti-rCsCP IgG reached 1:206,400 at 6 weeks after immunization (Fig. 4a, b).

After subcutaneous administration of $\mathrm{rCsCP}$, the specific IgG1 level remarkably increased at week $2\left(t_{(2)}=\right.$ 49.00, $P=10^{-9}$ ), while the IgG2a level showed obvious differences at week $4\left(t_{(2)}=64.56, P=10^{-9}\right)$, and both reached their peak at week 6 (Fig. 4c).

The specific IgG and IgG1/IgG2a isotype antibodies were induced by coat proteins extracted from B.s-CotC$C s \mathrm{CP}$ spores. The IgG level significantly increased from 


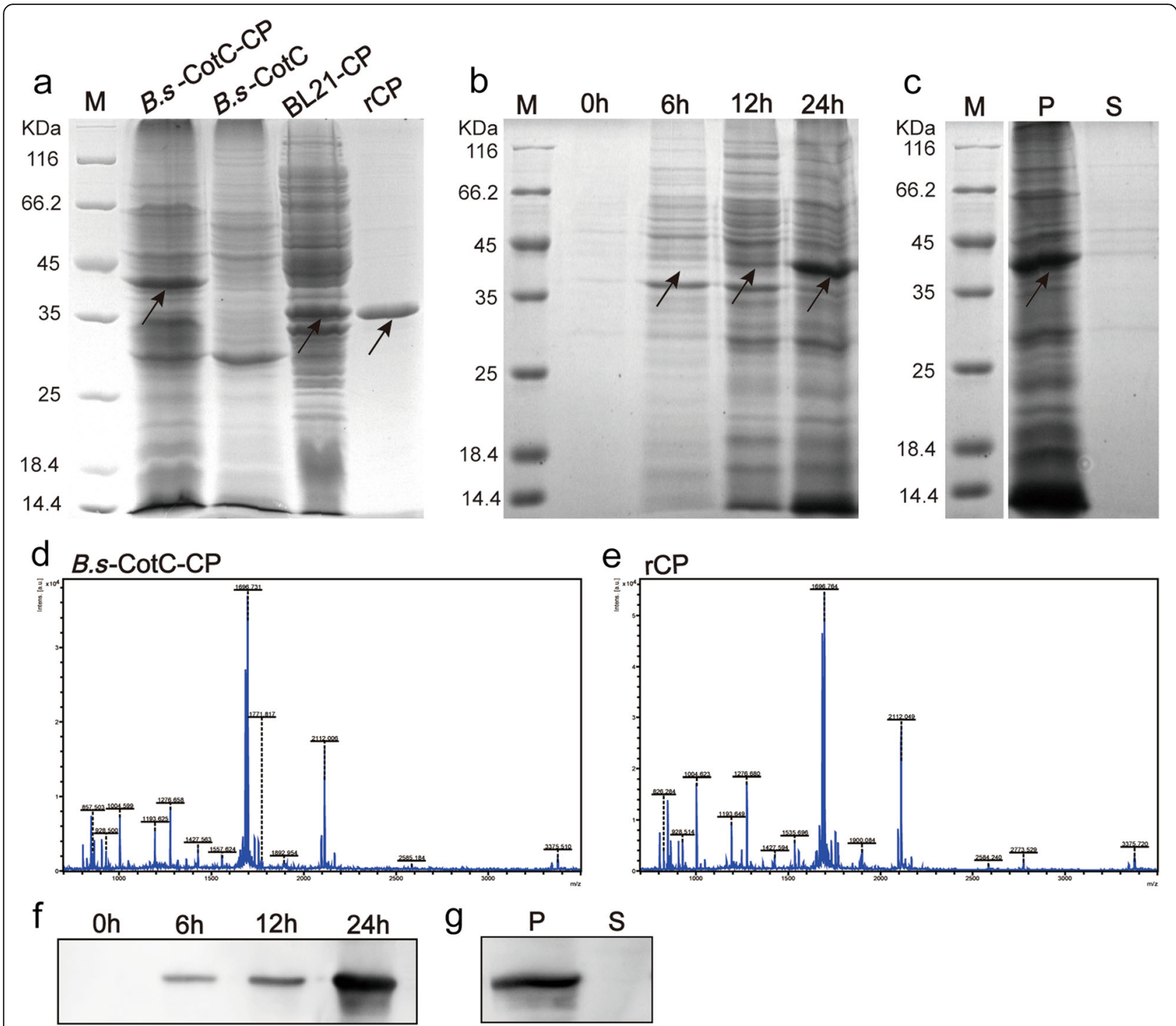

Fig. 2 Expression and identification of rCsCP and CotC-CsCP. a SDS-PAGE analysis of CsCP expressed in E. coli BL21 and B. subtilis spores. The molecular mass of CotC-CsCP fusion protein was approximately $43.8 \mathrm{kDa}$. Abbreviations: B.s-CotC-CP, WB600 containing pEB03-CotC-CsCP; B.s-CotC, WB600 harbouring pEB03-CotC; BL21-CP, BL21 harbouring pET28a-CSCP; rCP, purified rCSCP. $\mathbf{b}$ The expression of CotC-CsCP fusion protein at different sporulation times by $12 \%$ SDS-PAGE. c Total spore coat proteins extracted from recombinant spores (pEB03-CotC-CSCP) by SDS-PAGE analysis. d Identification of CotC-CSCP fusion protein by MS. e MALDI-TOF/TOF-MS analysis of purified rCsCP. $\mathbf{f}$ Expression identification of CotC-CSCP fusion protein at different sporulation times by Western blotting using rat anti-rCSCP serum. $\mathbf{g}$ Total coat proteins of pEB03-CotC-CsCP spore recognized by rat anti-rCsCP serum using Western blotting. Abbreviations: P, precipitation; S, supernatant

two to eight weeks after immunization, and the titre of specific IgG reached 1:1600 at week 6 (Fig. 4d, e). The specific IgG1 and IgG2a levels were significantly different compared with those in the PBS group at week 4 and week 6 (Fig. 4f).

\section{CsCP-specific antibodies in orally administered mice}

The specific IgG level in sera from B.s-CotC-CsCP group mice was significantly higher than of the PBS group after day $19\left(t_{(2)}=4.66, P=0.043\right)$ and reached peak titre on day 24 $\left(t_{(2)}=39, P=0.001\right)$. The level was significantly

Table 1 MALDI-TOF MS/MS identification results of the proteins from CotC-CsCP and rCsCP

\begin{tabular}{|c|c|c|c|c|c|c|c|c|}
\hline Sample & Protein name & MW (Da) & Protein PI & Protein score & $P$-value & Peptides matched & Swiss Prot & Accession number \\
\hline CotC-CsCP & C. sinensis cysteine protease precursor & 35,838 & 5.11 & 163 & $1 e^{-16}$ & 11 & 206x.1.A & gi|351693702 \\
\hline rCsCP & C. sinensis cysteine protease precursor & 35,383 & 5.11 & 159 & $2.5 e^{-16}$ & 11 & 206x.1.A & gi|351693702 \\
\hline
\end{tabular}




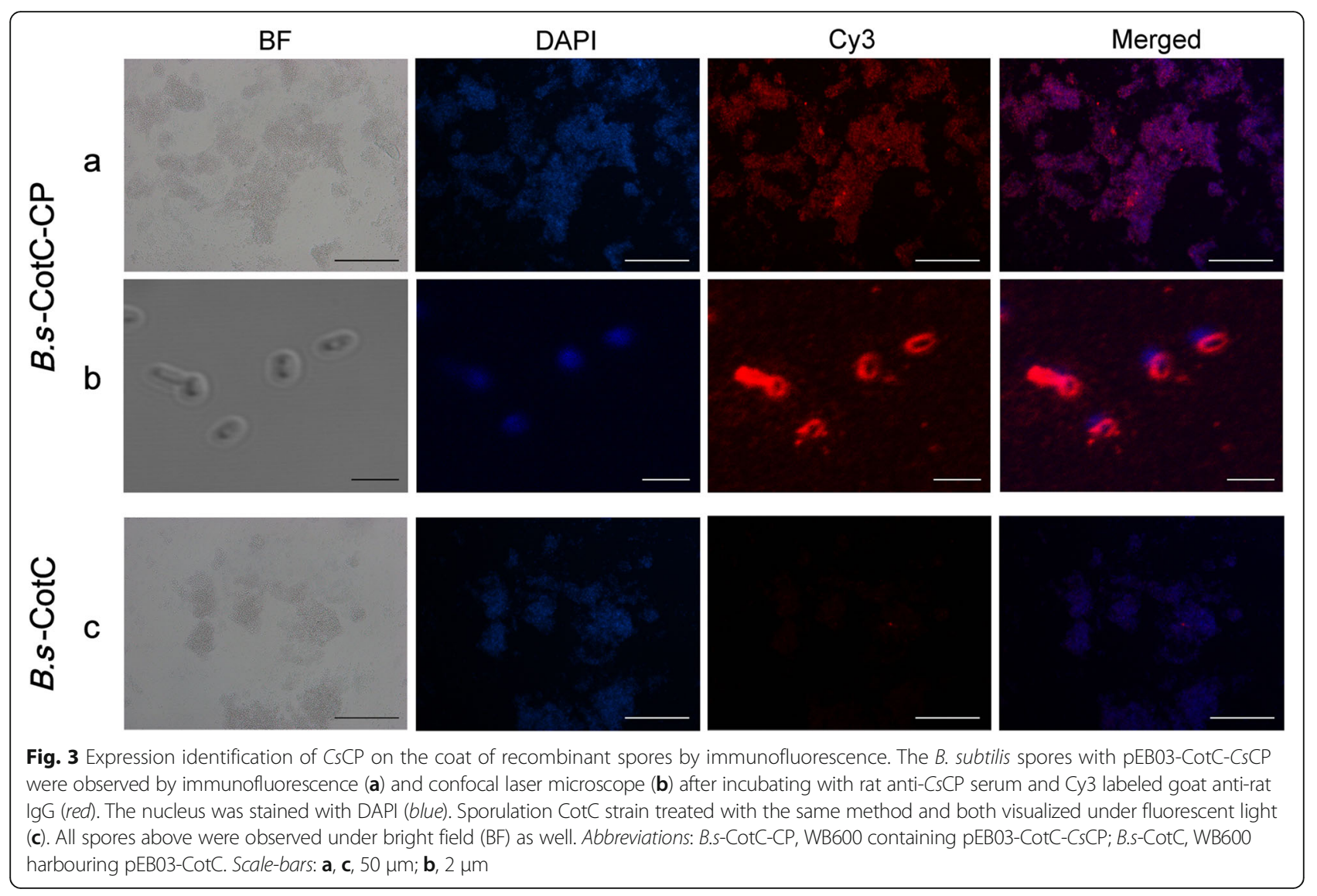

different than the BL21-CsCP group on day 24 and at week 6 (Fig. 5a). Specific IgG1 and IgG2a levels in the B.s-CotC$C s C P$ group were remarkably elevated at weeks 2 and 4 , respectively. The levels of IgG1 were significantly different between the B.s-CotC-CsCP and $\mathrm{BL} 21-\mathrm{CsCP}$ groups at week 4 (Fig. 5b). Specific IgA levels in the sera of the B.sCotC-CsCP mice were significantly higher than PBS group after week $4\left(t_{(2)}=5, P=0.038\right)$ (Fig. $\left.5 \mathrm{c}\right)$. At some points, the levels of anti-CsCP IgG, IgG1/IgG2a and IgA in serum significantly differed between BL21-CsCP group and PBS group. However, no significant difference was detected between the CotC group and the PBS group (Fig. 5).

Compared with the other three groups, specific IgG levels in intestinal mucus of B.s-CotC-CsCP group were relatively higher, and significantly elevated at week $6\left(t_{(2)}\right.$ $=4.33, P=0.049$ ) (Fig. 5d). Specific IgA levels in both intestinal mucus and bile of B.s-CotC-CsCP group were significantly higher than PBS group at week 4 and week 6 , respectively, and showed significant difference compared with BL21-CsCP group at the same time (Fig. 5e, f).

\section{IgA-secreting cells and mucosubstance in intestine}

After incubation with goat anti-mouse IgA and HRPprotein $A$, the sIgA cells in mouse intestine were observed by DAB staining (positive cells were indicated by dark brown). Compared with the other three groups, a large number of dark brown-stained cells were distributed in the enteraden and lamina propria regions of the mouse jejunum in the B.s-CotC-CsCP group $\left(P=10^{-9}\right.$; Fig. 6$)$.

After staining the slides with alcian blue and periodic acid-Schiff, many acidic mucins (marked in blue) were found in the intestines of the B.s-CotC and B.s-CotC$C s \mathrm{CP}$ groups. A small amount of acidic mucins were also found in the BL21-CsCP group, while copious neutral and alkaline mucus (indicated by amaranth) was present in the PBS group at week 4 (Fig. 7). Two weeks after the last immunization (week 6), a large amount of acidic mucosubstance was released from goblet cells in the B.s-CotC-CsCP group (Fig. 7).

\section{Biochemical index and histological analysis}

There was no significant difference in GPT/ALT and GOT/AST levels in serum amongst the groups (Fig. 8). In addition, no inflammatory injury was observed in the intestinal tissues of each group using haematoxylin-eosin staining (Additional file 3: Figure S3).

\section{Protective efficacy evaluation}

Six weeks post-challenge infection, livers of all mice were sent to Masson staining. As shown in Fig. 9, compared 

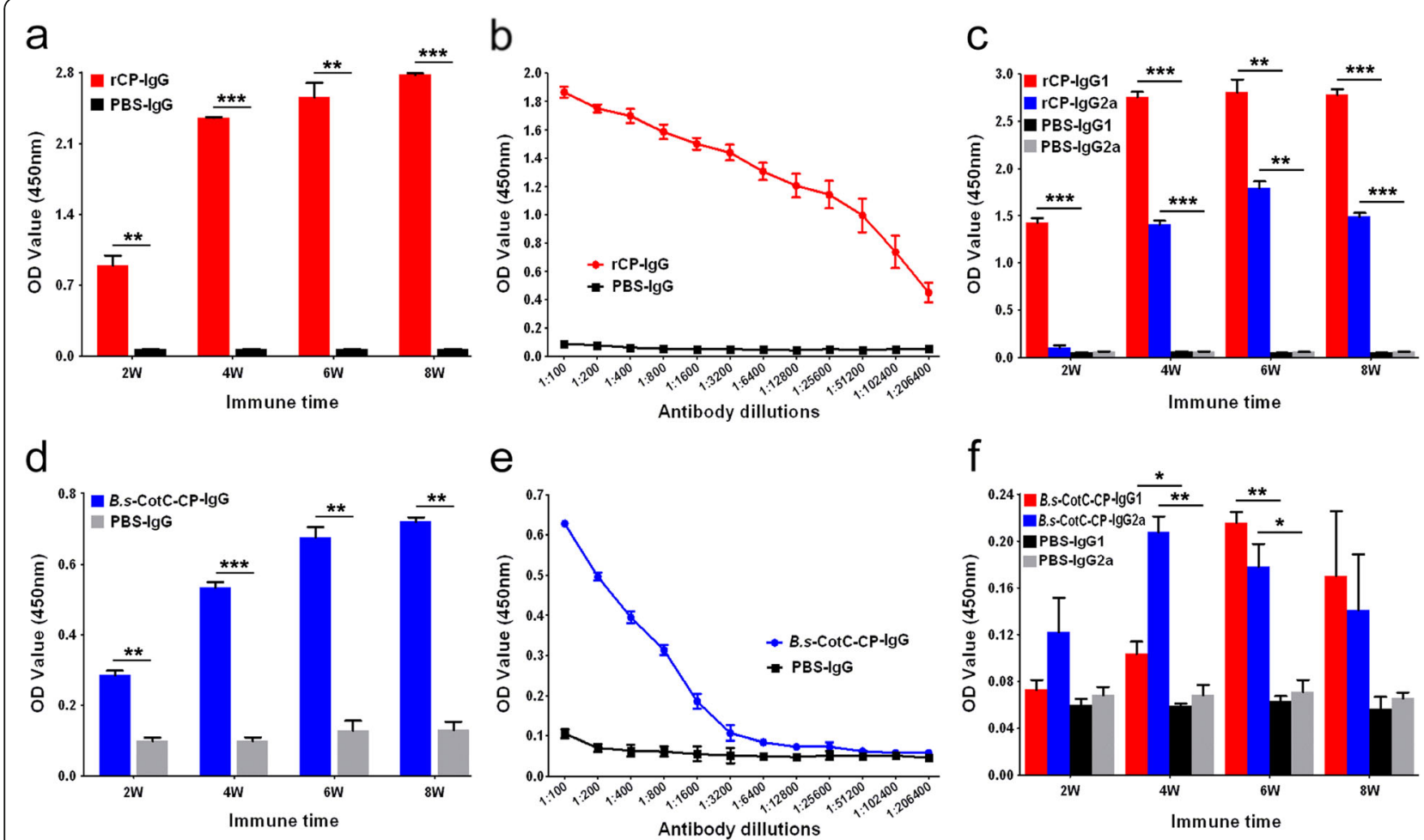

Fig. 4 Antibody titres of IgG and isotopes triggered by rCsCP and coat proteins of B.s-CotC-CsCP spores via subcutaneous immunization route. ELISA evaluation of the CSCP specific $\operatorname{lgG}$ a and $\operatorname{lgG} 1 / \lg$ G2a $\mathbf{c}$ levels in mouse sera after subcutaneous immunization with $\mathrm{rCSCP}$. $\mathbf{b}$ Antibody titres of $\lg G$ induced by rCsCP at week 6. The levels of CsCP specific $\lg \mathbf{d} \mathbf{d}$ and $\operatorname{lgG} 1 / \lg G 2 \mathrm{a} \mathbf{f}$ in the sera of mice subcutaneously immunized with spore coat proteins of B.s-CotC-CsCP. Antibody titres of IgG evoked by spore coat proteins of at week 6 were also assayed by ELISA e. Data were displayed as the mean \pm SD. ${ }^{*} P<0.05 ;{ }^{* * P}<0.01 ;{ }^{* *} P<0.001$. Abbreviations: B.s-CotC-CP, WB600 containing pEB03-CotC-CSCP; B.s-CotC, WB600 containing pEB03-CotC; rCP, purified rCsCP

with other groups, recombinant spores expressing $\mathrm{CsCP}$ administered mice had a lighter collagen deposition in the bile duct or hepatic parenchyma, and exhibited no obvious phenomenon of bile duct hyperplasia. Sections of adult worm were observed in some slides from mice of control groups but not in that from B.s-CotC-CsCP group. Ishak scores demonstrated that the degree of hepatic fibrosis could be significantly reduced after orally treated with spores of pEB03-CotC-CsCP (Fig. 9e).

\section{Discussion}

In the current study, we constructed a recombinant plasmid of pEB03-CotC-CsCP that could display original $C s C P$ on the coat of B. subtilis WB600 spore. The specific IgG was significantly induced by coat proteins extracted from B.s-CotC-CsCP spores after subcutaneous immunization. Compared with control groups, IgA levels in the serum, intestinal mucus and bile of the B.sCotC-CsCP orally treated mice increased remarkably. A larger number of IgA-secreting cells were observed in the enteraden and lamina propria regions of the mouse jejunum in the B.s-CotC-CsCP group. More acidic mucins and mucosubstance were found in the intestines of the B.s-CotC-CsCP group. Moreover, there was no significant difference in the GPT/ALT and GOT/AST levels in the sera from each group. No inflammatory injury was observed in the intestinal tissues of each group too.

The $\operatorname{CotC}-C s \mathrm{CP}$ fusion protein was abundantly expressed with extended induction time. SDS-PAGE and Western blotting showed that an obvious protein band at approximately $43.8 \mathrm{kDa}$ appeared in the B.s-CotC$\mathrm{Cs} \mathrm{CP}$ sample, and could be specifically recognized by anti-rCsCP rat sera (Fig. 2). In addition, we confirmed that $C s C P$ in the fusion protein presented by B. subtilis spores is similar to $\mathrm{rCs} C P$ purified from $E$. coil using mass spectrometry (Fig. 2). Immunofluorescence and confocal results clearly revealed that $\mathrm{CsCP}$ were enriched around the outer coat of spores (Fig. 3). This observation indicates that the stable spore delivery system was successfully exploited to express $C s C P$, and the CotC-CsCP fusion protein could directly contact the intestinal tract after oral administration.

In subcutaneously immunized mice, the antibody titres of $C s C P$ specific IgG reached 1:206,400 after three immunizations with $\mathrm{rCsCP}$ plus adjuvant (Fig. 4b). It demonstrated that $\mathrm{rCsCP}$ elicited high levels of specific 


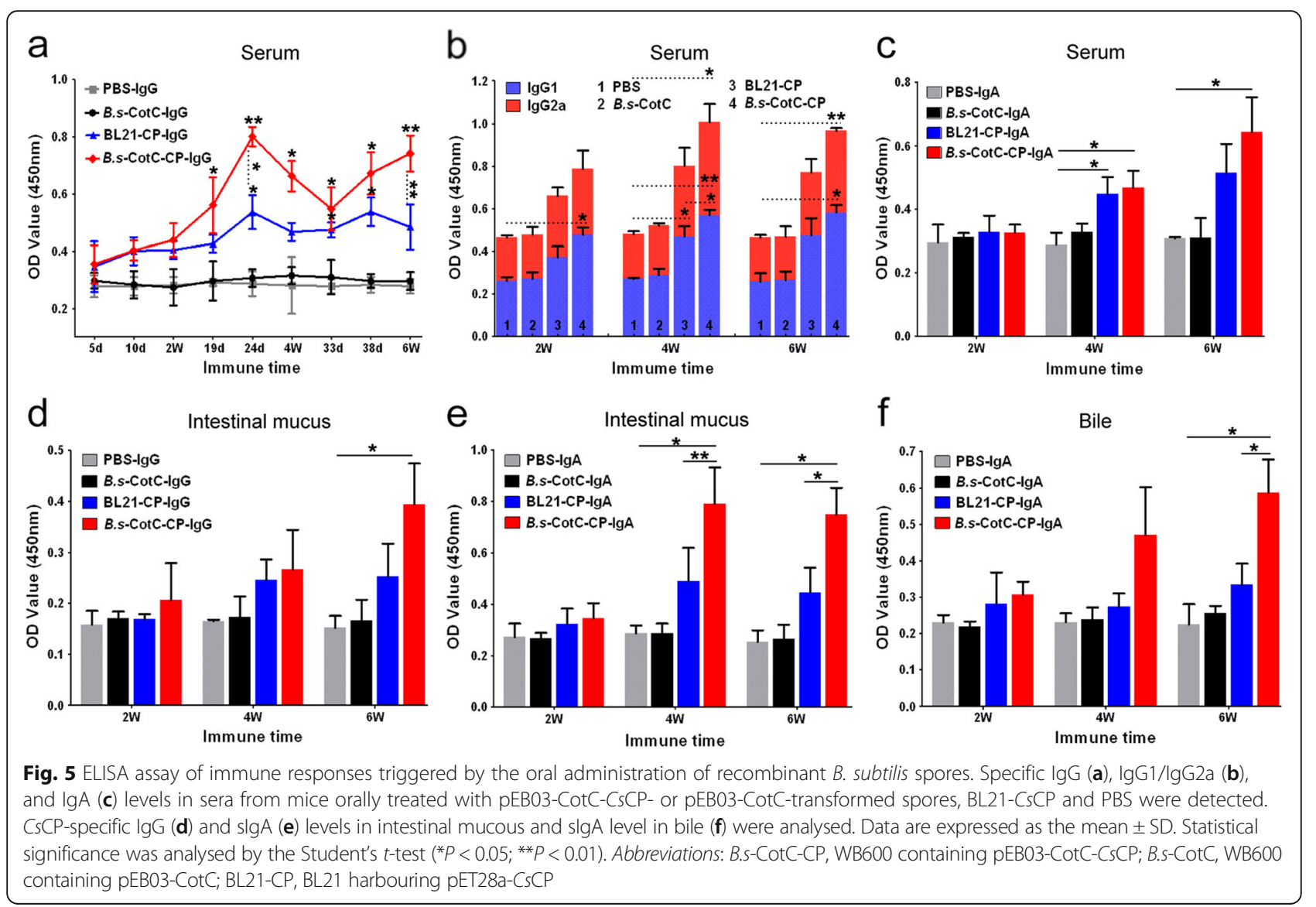

antibody rapidly and verified that $C s C P$ was of strong immunogenicity. When mice were administered with $\mathrm{rCs} C \mathrm{P}$, the specific IgG1 level in the serum significantly increased after week 2 and continued rising, while IgG2a levels were significantly elevated after week 4 (Fig. 4c). In mice treated with coat proteins extracted from the B.s-CotC-CsCP spore, the antibody titres of the specific IgG in serum reached 1:1600 at week 6. IgG1 and IgG2a levels significantly increased at weeks 4 and 6 . The IgG2a level at week 4 was higher than that of IgG1, but the levels were inversed at week 6 (Fig. 4d-f). Compared with $\mathrm{rCsCP}$, the specific antibody titres induced by the coat proteins of the B.s-CotC-CsCP spore were relatively lower. This result may have occurred because the coat proteins of the B.s-CotC-CsCP spore were isolated from the precipitation of spore lysate, which contained many other proteins in addition to $\mathrm{CsCP}$ (Fig. 2c). Additionally, an insoluble mixture might trigger a poorer immune response than soluble antigen with subcutaneous administration. The IgG1 antibody response generally represents helper $\mathrm{T}$ (Th) 2-mediated humoral immunity, while IgG2a represents Th1-mediated immunity [30, 31]. Our results showed that both the $\mathrm{rCsCP}$ and coat proteins of the B.s-CotC-CsCP spore could elicit Th1/Th2 combined immune responses in mice, but $\mathrm{rCsCP}$ induced a Th2 dominant response. There was no question as to the coat proteins of the B.s-CotC-CsCP spore. Collectively, these data demonstrated that $C s C P$ delivered by $B$. subtilis spores maintain the immunogenicity and effectively induce an immune response in mice.

Clonorchis sinensis metacercariae excyst in the duodenum of the definitive host and develop into larvae within a few minutes. Larvae rapidly migrate into bile ducts, and further maturate into adults $[1,2]$, so that during the invasion and parasitism, both the intestinal tract and the bile duct are important places for the development and growth of $C$. sinensis. This behaviour suggests that intervention measures applied in these two locations may be effective against $C$. sinensis infection. Cysteine proteases mainly distribute to the excretory bladder and the excretory granules of metacercariae. The cysteine protease family is one of the most abundantly expressed protease families in many digenetic trematode parasites (e.g. C. sinensis, Schistosoma japonicum, Clinostomum complanatum, Fasciola hepatica and Euclinostomum heterostomum) [18]. Moreover, cysteine protease is a key intrinsic endogenous enzyme that participates in the process of metacercariae excystment, invasion and immune modulation [18, 26, 32, 33]. Collectively, cysteine protease is considered to be a potential candidate 

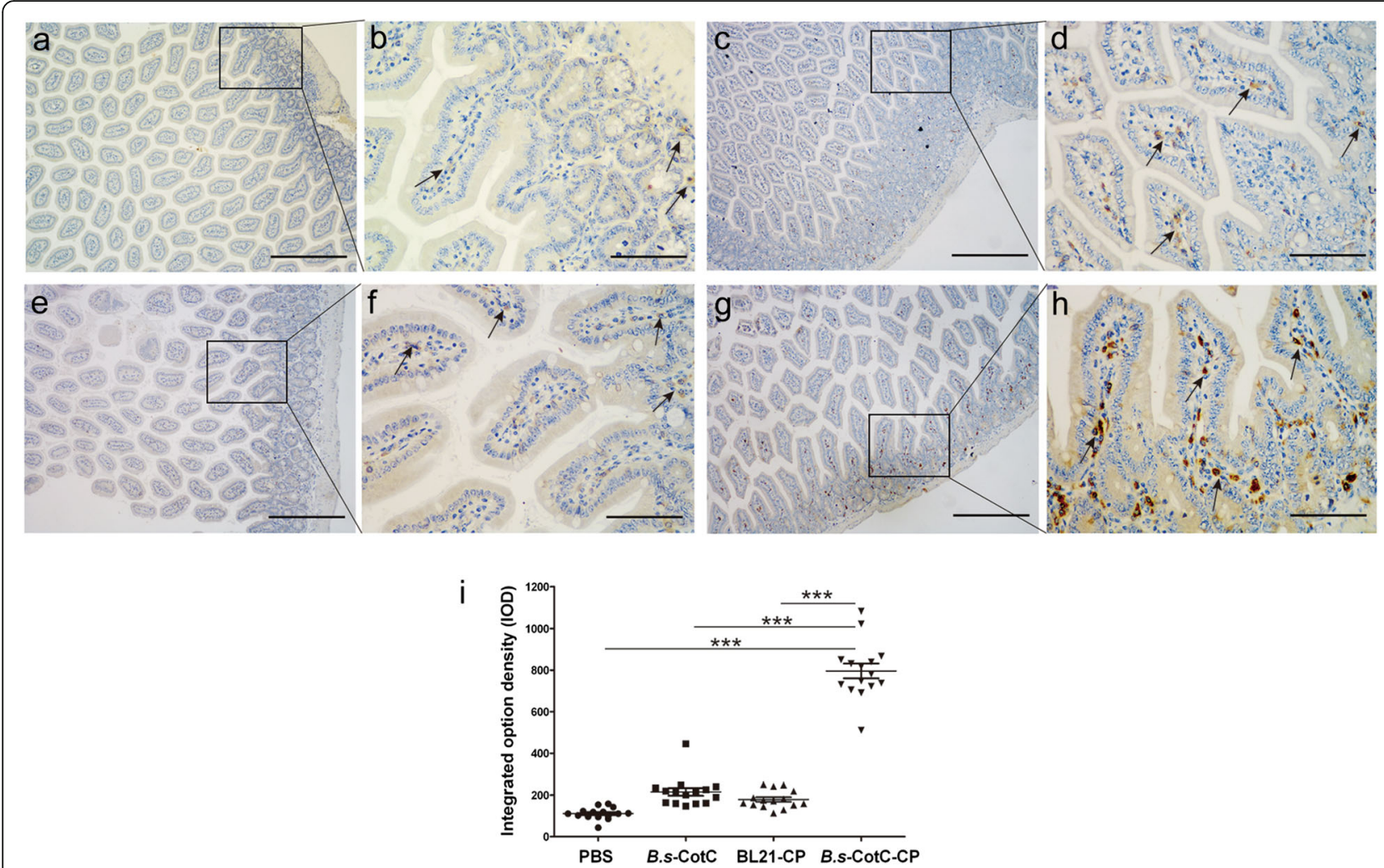

Fig. 6 Immunohistochemistry analysis of IgA-secreting cells in the intestinal epithelium of orally immunized mice. IgA-secreting cells were stained dark brown. The jejuna (approximately 5-7 $\mathrm{mm}$ ) of each group were isolated and submitted to immunohistochemical staining at week 4. Panels (a) and (b) represent PBS-treated mice. Panels (c) and (d) represent B.s-CotC orally administered mice. Panels (e) and (f) represent BL21-CsCP gavaged mice. Panels $(\mathbf{g})$ and $(\mathbf{h})$ represent mice orally administered with spores expressing CotC-CsCP. Scale-bars: $\mathbf{a}, \mathbf{c}, \mathbf{e}, \mathbf{g}, 200 \mu \mathrm{m} ; \mathbf{b}, \mathbf{d}, \mathbf{f}, \mathbf{h}$, $50 \mu \mathrm{m}$. The arrows indicate IgA-secreting cells. i Integrated option density (IOD) of IgA-secreting cells. ${ }^{* *} P<0.001$

molecule for the development of oral vaccine against $C$. sinensis infection.

Spores, the quiescent cell form of B. subtilis, are an ideal vehicle for delivery of heterologous antigens in the gastrointestinal tract because they are probiotics and resistant to extreme environments. They have previously served as effective adjuvants as well [15, 16, 34]. Bacillus subtilis spores (approximately $1.2 \mu \mathrm{m}$ in length) are well uptaken by $M$ cells, transported into Peyer's patches, processed by antigen-presenting cells, presented to B cells or $\mathrm{T}$ cells, and are transported to other gutassociated lymphoid tissues (GALTs) and systemic lymphoid tissues $[14,35]$. During this process, a series of antigen-specific immune responses are induced and abundant immune globulins are secreted by plasma cells [36]. As sIgA is the most abundantly produced immunoglobulin in the mammal mucosal system, it can serve as the first line of defence in protecting the intestinal epithelium from pathogenic microorganisms and enteric toxins $[37,38]$. In addition, sIgA promotes the clearance of pathogenic microorganisms and antigens by blocking their access to epithelial receptors, entrapping them in mucus, and facilitating their removal by peristaltic and mucociliary activities [38]. Our results show that CsCP recombinant spores evoked plenty of specific sIgA in intestinal mucosa and bile (Fig. 5e, f). A large number of IgA-secreting cells were observed in the jejunum of $B$. subtilis spores in orally administered mice, especially in the B.s-CotC-CsCP group (Fig. 6). IgA-secreting cells were primarily intestinal epithelial and lamina propria lymphocytes distributed in the enteraden and intestinal villus (Fig. 6). In addition, specific IgG arouse in intestinal mucus of B.s-CotC-CsCP group (Fig. 5d). Moreover, the levels of IgG, IgG1/IgG2a and IgA in the serum of the B.s-CotC-CsCP group were significantly higher than the control group (Fig. 5a-c). These results demonstrated that both local mucosal and systemic humoral immune responses were triggered by the oral administration of B.s-CotC-CsCP. Data also confirmed that the spores carried antigens that could trigger higher levels of specific antibody and be more suitable than E. coli for the development of oral vaccine vehicles carrying heterologous proteins.

Bacillus subtilis spores are probiotic microorganism that are used as a food supplement and can promote intestinal health (e.g. aiding in digestion and being 

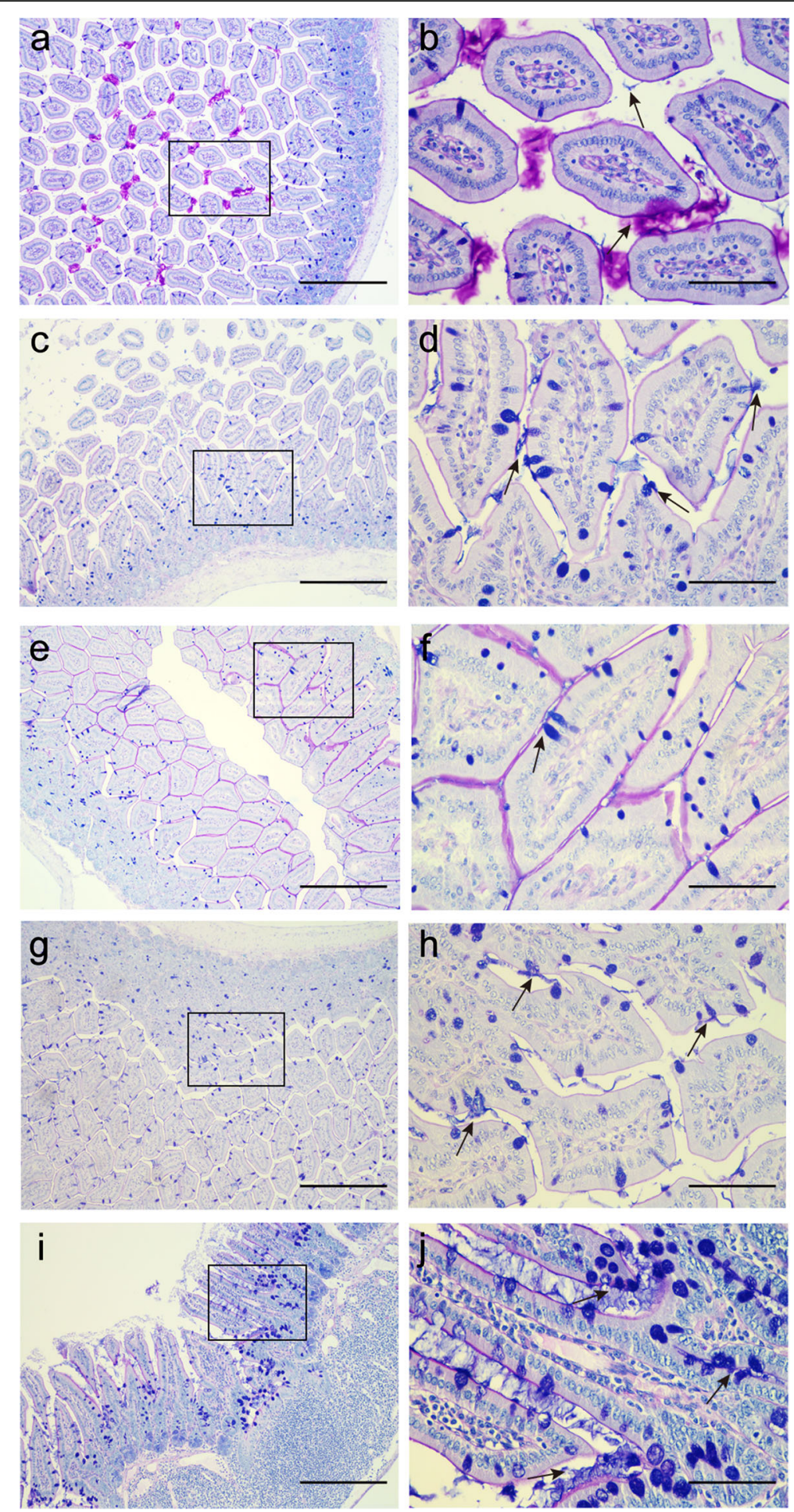

Fig. 7 AB-PAS stain of mucins in the intestinal epithelium of oral administration mice. Jejunum tissue sections of each group were collected, fixed, and stained with AB-PAS. Acid mucins were dyed to blue, neutral mucin were dyed red, and the alkaline and neutral mixed mucins were dyed amaranth. Panels a-b, $\mathbf{c}-\mathbf{d}$, e-f and $\mathbf{g}-\mathbf{h}$ indicate PBS, B.s-CotC, BL21-CsCP and B.s-CotC-CsCP orally administered groups at week 4, respectively. Panels (i) and (j) show the B.s-CotC-CsCP group treated at week 6. Scale-bars: $\mathbf{a}, \mathbf{c}, \mathbf{e}, \mathbf{g}, \mathbf{i}, 200 \mu \mathrm{m} ; \mathbf{b}, \mathbf{d}, \mathbf{f}, \mathbf{h}, \mathbf{j}, 50 \mu \mathrm{m}$. The arrows indicate acidic mucins secreted by goblet cells 


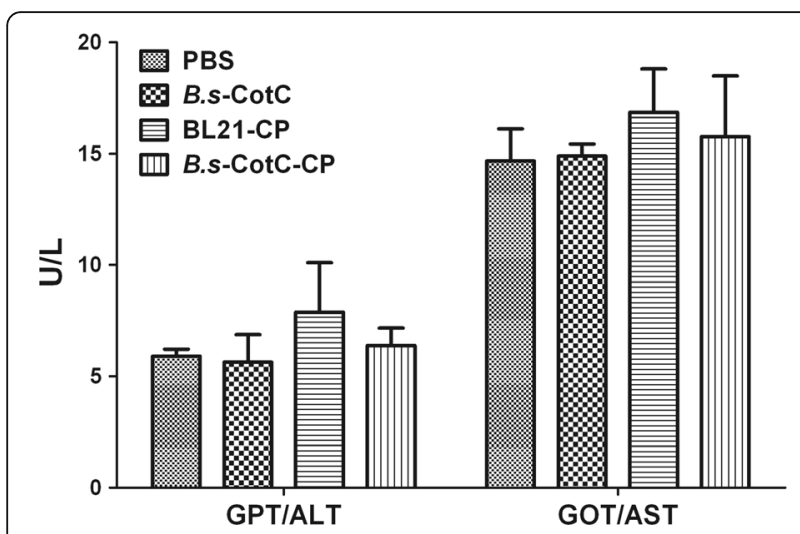

Fig. 8 The activity of GPT/ALT and GOT/AST in the serum of oral immunization mice. The serum in PBS, B.s-CotC, BL21-CsCP and B.sCotC-CsCP groups was collected at week 6 . The levels of GPT/ALT and GOT/AST were analysed. Abbreviations: B.s-CotC-CP, WB600 containing pEB03-CotC-CSCP; B.s-CotC, WB600 of pEB03-CotC; BL21-CP, BL21 harbouring pET28a-CSCP beneficial for the balance of intestinal microbiota and regulation of intestinal mucus environment) [10, 39]. The mucus layer overlaying the epithelium secreted by the goblet cells of the gastrointestinal tract serves as the first line of defence against physical and chemical injury from luminal contents and pathogens and provides a natural habitat for commensal microbiota [40-42]. Mucins, the major component of mucus, can be broadly categorized into neutral and acidic chemotypes. High levels of acidic mucins such as sialomucin and sulfomucin reportedly coincide with maturation of intestinal barrier function and have been implicated in the protection of the mucosa from pathogens [43, 44]. Microbiota and microbial products, meanwhile, can modulate the synthesis and secretion of mucins, either by directly activating diverse signalling cascades or through bioactive factors generated by epithelial and lamina propria cells [42]. In our experiments, B. subtilis spores could
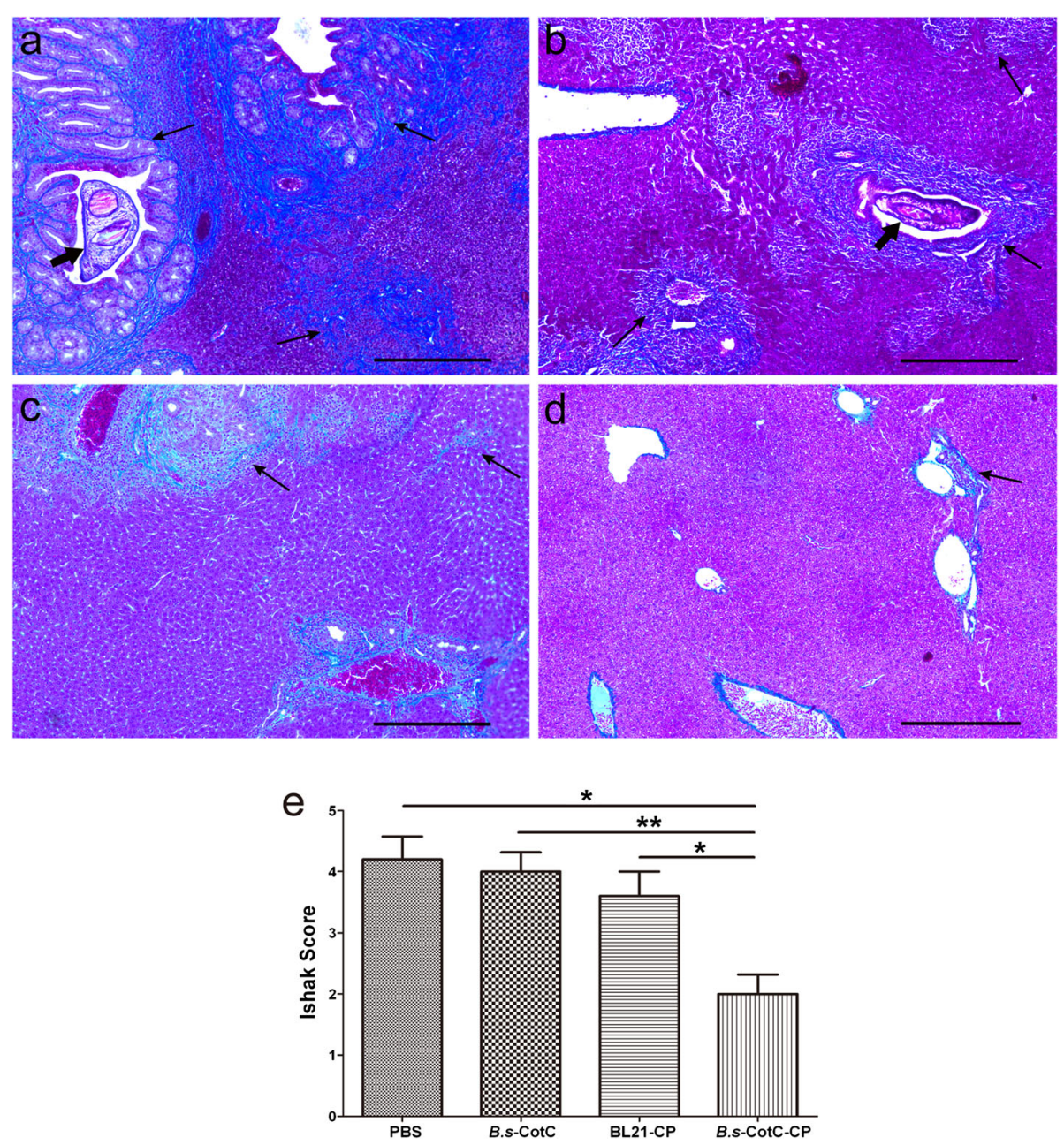

Fig. 9 Pathological changes of livers from challenge infection mice. Liver sections of each group were stained with Masson's trichrome. Collagen fibers are shown in blue. Panels a-d show mice orally treated with PBS, B.s-CotC, BL21-CsCP and B.s-CotC-CsCP, respectively. Panel (e) provides the statistic of Ishak scores in liver sections from each mouse. ${ }^{*} P<0.05 ;{ }^{* *} P<0.01$. The thick arrows indicate the adult worms of $C$. sinensis, and the thin arrows indicate collagen deposition in livers. Scale-bars: $200 \mu \mathrm{m}$ 
provoke an increase in acidic intestinal mucus (Fig. 7). Thus, B. subtilis spores may enhance resistance to pathogens, and guarantee intestinal health.

It has been documented that B. subtilis spores are safe food probiotics. No signs of toxicity or virulence were found in vivo assessments, and no side effects appeared in the liver function of the rats after oral treatment $[10,16]$. Our findings also confirmed that there was no damage to the liver or intestine of mice after oral administration of $B$. subtilis spores (Fig. 8; Additional file 3: Figure S3). Challenge experiment verified that oral administration of $B$. subtilis spores expressing $\mathrm{CsCP}$ could effectively fight against metacercaria $C$. sinensis infection by reducing the process of liver fibrosis in mice (Fig. 9).

\section{Conclusions}

We confirmed the excellent immunogenicity of $C s \mathrm{CP}$ and B. subtilis spores as an oral delivery vehicle that maintained their original immunogenicity by displaying $C_{s} \mathrm{CP}$ on the spore surface. Both local and systemic specific immune responses were elicited by the oral administration of B. subtilis spores expressing CsCP. Moreover, the spores could effectively promote intestinal health by inducing the secretion of acidic mucins with no side effects to the liver or intestine. Effective protection against $C$. sinensis was triggered after oral administration of spores expressing $C s \mathrm{CP}$. This study is a cornerstone for the development of antiparasitic agents or vaccines against clonorchiasis based on $B$. subtilis spores expressing $C s \mathrm{CP}$ on the surface.

\section{Additional files}

Additional file 1 Figure S1. Genetic engineering of B. subtilis spores and E. coil expressing CsCP. (DOC $977 \mathrm{~kb}$ )

Additional file $\mathbf{2}$ Figure S2. Prokaryotic expression and purification of the recombinant CSCP. (DOC $2128 \mathrm{~kb}$ )

Additional file $\mathbf{3}$ Figure S3. Histopathological features of the jejuna from spores immunized mice. (DOC $5180 \mathrm{~kb}$ )

\section{Abbreviations}

B. s.: Bacillus subtilis; CCA: Cholangiocarcinoma; CotC: Coat protein C, one of coat proteins of $B$. subtilis spore; CsCP: Cysteine protease of $C$. sinensis; DALYs: Disability adjusted life years; ESPs: Excretory-secretory products.; GOT/AST: Glutamic oxaloacetic transaminase/aspartate aminotransferase; GPT/ALT: Glutamic pyruvic transaminase/alanine aminotransferase; rCsCP: Recombinant CsCP protein

\section{Acknowledgments}

We would like to acknowledge the platform of Proteomics Center from Zhongshan School of Medicine, Sun Yat-sen University providing the MS assay in this work.

\section{Funding}

This work was supported by the Science and Technology Planning Project of Guangdong Province (No. 2013 B010404010 and No. 2014B020203001), the National Key Basic Research and Development Project of China (973 project; No.2010CB530000), Innovative Research Teams Project of South Wisdom Valley, Shunde, Guangdong province (2013CXTD03), and Young Teachers'
Cultivation Project of Basic Scientific Research Service Fee in Colleges and Universities (No.16ykpy06)

\section{Availability of data and materials}

The datasets generated during and/or analysed during the current study are available in the GenBank database repository (https://www.ncbi.nlm.nih.gov/ nuccore/JN655695).

\section{Authors' contributions}

Conceived and designed the experiments: ZT, MS, YH, JX and XY. Performed the experiments: ZT and MS. Analyzed the data: ZT, MS, TC and PR. Contributed reagents/materials/analysis tools: ZT, TC, HS, HQ, ZL, LZ, JY, HJ, XZ and XL. Wrote the paper: $\mathrm{ZT}, \mathrm{YH}$ and $\mathrm{XY}$. All authors read and approved the final version of the manuscript.

\section{Competing interests}

The authors declare that they have no competing interests.

Consent for publication

Not applicable.

Ethics approval and consent to participate

All mice used in this study were purchased from Animal Center of Sun Yat-Sen University and raised carefully in accordance with National Institutes of Health on animal care and the ethical guidelines. All animal studies were approved by the Sun Yat-Sen University Institutional Animal Care and Use Committee (Permit Numbers: SYXK (Guangdong) 2010-0107).

Received: 1 July 2016 Accepted: 4 December 2016

Published online: 19 December 2016

\section{References}

1. Lun ZR, Gasser RB, Lai DH, Li AX, Zhu XQ, Yu XB, et al. Clonorchiasis: a key foodborne zoonosis in China. Lancet Infect Dis. 2005;5(1):31-41.

2. Qian MB, Utzinger J, Keiser J, Zhou XN. Clonorchiasis. Lancet. 2016; 387(10020):800-10.

3. Wang $X$, Chen W, Huang $Y$, Sun J, Men J, Liu H, et al. The draft genome of the carcinogenic human liver fluke Clonorchis sinensis. Genome Biol. 2011; 12(10):R107.

4. Fürst T, Keiser J, Utzinger J. Global burden of human food-borne trematodiasis: a systematic review and meta-analysis. Lancet Infect Dis. 2012;12(3):210-21.

5. Streatfield SJ. Mucosal immunization using recombinant plant-based oral vaccines. Methods. 2006:38(2):150-7.

6. Davitt CJ, Lavelle EC. Delivery strategies to enhance oral vaccination against enteric infections. Adv Drug Deliv Rev. 2015;91:52-69.

7. Patel H, Yewale C, Rathi MN, Misra A. Mucosal immunization: a review of strategies and challenges. Crit Rev Ther Drug Carrier Syst. 2014;31(4):273-303.

8. Ciabattini A, Parigi R, Isticato R, Oggioni MR, Pozzi G. Oral priming of mice by recombinant spores of Bacillus subtilis. Vaccine. 2004;22(31-32):4139-43.

9. Amuguni $\mathrm{H}$, Tzipori S. Bacillus subtilis: a temperature resistant and needle free delivery system of immunogens. Hum Vaccin Immunother. 2012;8(7): 979-86.

10. Hong HA, Huang JM, Khaneja R, Hiep LV, Urdaci MC, Cutting SM. The safety of Bacillus subtilis and Bacillus indicus as food probiotics. J Appl Microbiol. 2008;105(2):510-20.

11. le Duc H, Hong HA, Cutting SM. Germination of the spore in the gastrointestinal tract provides a novel route for heterologous antigen delivery. Vaccine. 2003:21(27-30):4215-24.

12. Knecht $L D$, Pasini $P$, Daunert $S$. Bacterial spores as platforms for bioanalytical and biomedical applications. Anal Bioanal Chem. 2011;400(4):977-89.

13. Uyen NQ, Hong HA, Cutting SM. Enhanced immunisation and expression strategies using bacterial spores as heat-stable vaccine delivery vehicles. Vaccine. 2007;25(2):356-65

14. Zhou Z, Xia H, Hu X, Huang Y, Li Y, Li L, et al. Oral administration of a Bacillus subtilis spore-based vaccine expressing Clonorchis sinensis tegumental protein $22.3 \mathrm{kDa}$ confers protection against Clonorchis sinensis. Vaccine. 2008; 26(15):1817-25.

15. Wang X, Chen W, Tian Y, Mao Q, Lv X, Shang M, et al. Surface display of Clonorchis sinensis enolase on Bacillus subtilis spores potentializes an oral vaccine candidate. Vaccine. 2014;32(12):1338-45. 
16. Yu J, Chen T, Xie Z, Liang P, Qu H, Shang M, et al. Oral delivery of Bacillus subtilis spore expressing enolase of Clonorchis sinensis in rat model: induce systemic and local mucosal immune responses and has no side effect on liver function. Parasitol Res. 2015;114(7):2499-505.

17. Trap C, Boireau P. Proteases in helminthic parasites. Vet Res. 2000;31(5):461-71.

18. Shareef PA, Abidi SM. Cysteine protease is a major component in the excretory/secretory products of Euclinostomum heterostomum (Digenea: Clinostomidae). Parasitol Res. 2014;113(1):65-71.

19. Lv X, Chen W, Wang X, Li X, Sun J, Deng C, et al. Molecular characterization and expression of a cysteine protease from Clonorchis sinensis and its application for serodiagnosis of clonorchiasis. Parasitol Res. 2012;110(6):2211-9.

20. Gonzales Santana B, Dalton JP, Vasquez Camargo F, Parkinson M, Ndao M. The diagnosis of human fascioliasis by enzyme-linked immunosorbent assay (ELISA) using recombinant cathepsin L protease. PLoS Negl Trop Dis. 2013; 7(9):e2414. doi:10.1371/journal.pntd.0002414

21. El-Ahwany E, Rabia I, Nagy F, Zoheiry M, Diab T, Zada S. Protective role of purified cysteine proteinases against Fasciola gigantica infection in experimental animals. Korean J Parasitol. 2012;50(1):45-51.

22. Zimic M, Pajuelo M, Rueda D, López C, Arana Y, Castillo Y, et al. Utility of a protein fraction with cathepsin L-Like activity purified from cysticercus fluid of Taenia solium in the diagnosis of human cysticercosis. Am J Trop Med Hyg. 2009;80(6):964-70.

23. Baig S, Damian RT, Morales-Montor J, Ghaleb A, Baghdadi A, White Jr AC. Protection from murine cysticercosis by immunization with a parasite cysteine protease. Microbes Infect. 2006;8(12-13):2733-5.

24. Loukas A, Bethony JM, Williamson AL, Goud GN, Mendez S, Zhan B, et al. Vaccination of dogs with a recombinant cysteine protease from the intestine of canine hookworms diminishes the fecundity and growth of worms. J Infect Dis. 2004;189(10):1952-61.

25. Loukas A, Dowd AJ, Prociv P, Brindley PJ. Purification of a diagnostic, secreted cysteine protease-like protein from the hookworm Ancylostoma caninum. Parasitol Int. 2000;49(4):327-33.

26. Li S, Chung YB, Chung BS, Choi MH, Yu JR, Hong ST. The involvement of the cysteine proteases of Clonorchis sinensis metacercariae in excystment. Parasitol Res. 2004;93(1):36-40.

27. Qu H, Xu Y, Sun H, Lin J, Yu J, Tang Z, et al. Systemic and local mucosal immune responses induced by orally delivered Bacillus subtilis spore expressing leucine aminopeptidase 2 of Clonorchis sinensis. Parasitol Res. 2014;113(8):3095-103.

28. Nicholson WL, Setlow P. Sporulation, germination and outgrowth. In: Harwood CR, Cutting SM, editors. Molecular Biological Methods for Bacillus. Chichester, UK: John Wiley \& Sons Ltd.; 1990. p. 391-450.

29. Ishak K, Baptista A, Bianchi L, Callea F, De Groote J, Gudat F, et al. Histological grading and staging of chronic hepatitis. J Hepatol. 1995;22(6):696-9.

30. Ebrahimpoor S, Pakzad SR, Ajdary S. IgG1 and IgG2a profile of serum antibodies to Leishmania major amastigote in BALB/C and C57BL/6Mice. Iran J Allergy Asthma Immunol. 2013;12(4):361-7.

31. Yanase N, Toyota H, Hata K, Yagyu S, Seki T, Harada M, et al. OVA-bound nanoparticles induce OVA-specific lgG1, lgG2a, and lgG2b responses with low lgE synthesis. Vaccine. 2014;32(45):5918-24.

32. Chung YB, Kim TS, Yang HJ. Early cysteine protease activity in excretory bladder triggers metacercaria excystment of Paragonimus westermani. J Parasitol. 2005;91(4):953-4.

33. Villalobo E, Moch C, Fryd-Versavel G, Fleury-Aubusson A, Morin L. Cysteine proteases and cell differentiation: excystment of the ciliated protist Sterkiella histriomuscorum. Eukaryot Cell. 2003;2(6):1234-45.

34. de Souza RD, Batista MT, Luiz WB, Cavalcante RC, Amorim JH, Bizerra RS, et al. Bacillus subtilis spores as vaccine adjuvants: further insights into the mechanisms of action. PLoS One. 2014;9(1):e87454. doi:10.1371/journal.pone.0087454.

35. Tavares Batista M, Souza RD, Paccez JD, Luiz WB, Ferreira EL, Cavalcante RC, et al. Gut adhesive Bacillus subtilis spores as a platform for mucosal delivery of antigens. Infect Immun. 2014;82(4):1414-23.

36. Lamichhane A, Azegamia T, Kiyonoa $\mathrm{H}$. The mucosal immune system for vaccine development. Vaccine. 2014;32(49):6711-23.

37. Macpherson AJ, Hunziker L, McCoy K, Lamarre A. IgA responses in the intestinal mucosa against pathogenic and non-pathogenic microorganisms. Microbes Infect. 2001;3(12):1021-35

38. Mantis NJ, Rol N, Corthésy B. Secretory lgA's complex roles in immunity and mucosal homeostasis in the gut. Mucosal Immunol. 2011;4(6):603-11.
39. de Moreno de LeBlanc A, LeBlanc JG. Effect of probiotic administration on the intestinal microbiota, current knowledge and potential applications. World J Gastroenterol. 2014;20(44):16518-28.

40. Belley A, Keller K, Göttke M, Chadee K. Intestinal mucins in colonization and host defense against pathogens. Am J Trop Med Hyg. 1999;60 Suppl 4:10-5.

41. Barnett AM, Roy NC, McNabb WC, Cookson AL. The interactions between endogenous bacteria, dietary components and the mucus layer of the large bowel. Food Funct. 2012;3(7):690-9.

42. Kim YS, Ho SB. Intestinal goblet cells and mucins in health and disease: recent insights and progress. Curr Gastroenterol Rep. 2010;12(5):319-30.

43. Soga K, Yamauchi J, Kawai Y, Yamada M, Uchikawa R, Tegoshi T, et al. Alteration of the expression profiles of acidic mucin, sialytransferase, and sulfotransferases in the intestinal epithelium of rats infected with the nematode Nippostrongylus brasiliensis. Parasitol Res. 2008;103(6):1427-34.

44. Forder RE, Howarth GS, Tivey DR, Hughes RJ. Bacterial modulation of small intestinal goblet cells and mucin composition during early posthatch development of poultry. Poult Sci. 2007:86(11):2396-403.

\section{Submit your next manuscript to BioMed Central and we will help you at every step:}

- We accept pre-submission inquiries

- Our selector tool helps you to find the most relevant journal

- We provide round the clock customer support

- Convenient online submission

- Thorough peer review

- Inclusion in PubMed and all major indexing services

- Maximum visibility for your research

Submit your manuscript at www.biomedcentral.com/submit
) Biomed Central 